\title{
Post-Stroke Depression
}

\section{${ }^{1}$ Department of \\ Neurology, University \\ Cinical Centre Tuzla and \\ University of Tuzla, Tuzla, \\ Bosnia and Herzegovina \\ ${ }^{2}$ Centre of Neurology of \\ the Primary Health Care \\ Centre with Polyclinic \\ Department „Dr Mustafa \\ Sehovic", University of \\ Tuzla, Tuzla, Bosnia and Herzegovina \\ ${ }^{3}$ Department of Hygiene and Epidemiology, \\ University Cinical Centre \\ Tuzla and University of \\ Tuzla, Tuzla, Bosnia and \\ Herzegovina}

\section{Corresponding author:}

Suljo Kunic, MD. Centre of neurology of the Primary health centre Tuzla, University of Tuzla, Tuzla, Bosnia and Herzegovina. E-mail: suljo.kunic@hotmail. com. ORCID ID: http:// www.orcid.org: 00000001-7900-1222.

\section{Omer C. Ibrahimagic ${ }^{1}$, Dzevdet Smajlovic ${ }^{1}$, Suljo Kunic ${ }^{2}$, Zikrija Dostovic ${ }^{1}$, Amer Custovic $^{3}$, Aida Sehanovic ${ }^{1}$, Biljana Kojic ${ }^{1}$}

\section{ABSTRACT}

Introduction: The depression is a common mental disorder, especially after a stroke, which further aggravates the recovery. Aim: To analyze depression within 48 hours and fifteen days after ischemic stroke in relation to gender and location (brain hemisphere and brain circulation). Methods: We analyzed 40 patients $(65.3 \pm 10.3$ years), half of them were women. Mean age of women was $66.35 \pm 7.31$ years and men $64.2 \pm 12.68$ years $(p=0.5)$. Ischemic stroke was verified by computed tomography. Levels of depression were measured with self-estimated Zung's scale. On the tests, score of 50 and higher verified depression. Criteria made by Domasio were used to determine location of the IS. Results: Mean value on depression scale in acute phase of ischemic stroke was $46.85 \pm$ 8.6 and in subacute phase $43.4 \pm 8$ ( $p=0.06)$. In 19 ( $47.5 \%$ ) patients ( $55 \%$ of women, $40 \%$ of men; $p=0.3$ ) depression was found during the first and in 10 (25\%) patients (35\% of women, $15 \%$ of men; $p=0.06$ ) during the second evaluation $(p<0.019)$. Mean value on depression in acute phase of illness in women was $49.1 \pm 7.38$, as well as in men $44.6 \pm 9.22(p=0.088)$ and in subacute phase in women $45.25 \pm 8.04$, as well as in men $41.5 \pm 7.75(p=0.16)$. Concerning location of ischemic stroke, there were no significant differences in levels of depression. Conclusion: Number of patients with post-stroke depression is significantly lower in subacute phase of ischemic stroke. Although the number of depressive women and their depression scores are higher, gender differences are not statistically significant. There is no correlation between post-stroke depression and location of lesion in acute and subacute phase of illness.

Keywords: Cerebrum, Depression, Sex, Stroke.

\section{INTRODUCTION}

Ischemic stroke (IS) is a rapid clinical sign of development (focal or global) of brain function disorder with symptoms lasting 24 hours or longer, or leading to death, with no clear other cause but blood vessel destruction (1). Accord- ing to the report of World Health Organization (WHO), there are 15 million people suffering from stroke every year (2). Unfortunately, mood and emotional disturbances are frequent problems in stroke survivors (3). They are distressing for patients and their caregivers, with negative influence on quality of life both of them. Mood disorders include post-stroke depression (PSD), post-stroke anxiety, post-stroke emotional incontinence, post-stroke anger proneness, as well as post-stroke fatigue $(4,5)$. Emotional disturbances are not apparent and therefore are often unnoticed by busy clinicians. Their phenomenology, predicting factors, and pathophysiology have been under-studied and under-recognized (6). According to few older investigations, PSD is present in $20-55 \%$ of the patients in acute phase of IS $(7,8,9)$.

\section{AlM}

Aim of our study was to analyze rising of depression in acute and subacute phase of IS in relation to gender and location of the brain lesion (brain hemisphere/brain circulation).

\section{METHODS}

We analyzed 40 consecutively recruited patients (gender ratio 20:20) hospitalized for the first IS at Department of Neurology, University Clinical Center Tuzla, Bosnia and Herzegovina. Mean age of patients was $65.3 \pm 10.3$ years (Women 66.35 $\pm 7.31 /$ Men 64.2 \pm 12.68$)(p=0.5)$. Exclusion criteria for our experimental group were: loss of consciousness, aphasia, neglect, history of mental disorders and previous IS. In our experimental groups, IS was verified after neurological examination and computed tomography of the brain (for some of them we used magnetic resonance imaging). Our patients, due to their current neurological status, were able to answer on 20 questions contained in self-estimated depression scale made by Zung (10). This scale was compared to other screening methods in 
geriatric stroke patients and assessed with a high positive predictive value (11). Level of depression was measured within 48 hours and fifteen days after IS (acute and subacute phase). Score of 50 and higher was verification of some kind of depression. Criteria made by Domasio were used to determine location of IS (12). Our investigation was conducted in accordance with the World Medical Declaration of Helsinki (Ethical principles for medical research involving human subjects from 1975 and its amendments), with the full understanding and the written informed consent of the participants. The Ethical Committee of University Clinical Center Tuzla has approved experiments in our study. Standard statistical test have been used: mean value, t-test and Chi-square test ( $\chi^{2}$-test). A statistical significance level of 95\% ( $p<0.05)$ was considered to be the limit for all tests.

\section{RESULTS}

Mean value on Zung's scales in acute phase of IS was $46.85 \pm 8.6(29-65)$ and it was verified in 19 (47.5\%) patients. In subacute phase of IS mean value was $43.4 \pm 8$ (26-59), what was almost significantly different to level of depression within 48 hours $(\mathrm{p}=0.06)$. Depression persisted during the second evaluation in $10(25 \%)$ patients $(\mathrm{p}<0.019)$ (Figure 1).

Mean value on Zung's scales in acute phase of IS in women was 49.1 \pm 7.38 (38-65) and inmen44.6 $\pm 9.22(29-59)(\mathrm{p}=0.088)$. Depression was registered in 11 women (55\%) and 8 men (40\%) $(\mathrm{p}=0.3)$. Mean value on Zung's scales in subacute phase of IS in women was $45.25 \pm 8.04$ (31-59) and in men $41.5 \pm 7.75$ (26-55) $(p=0.16)$. Depression was registered in 7 women $(35 \%)$ and 3

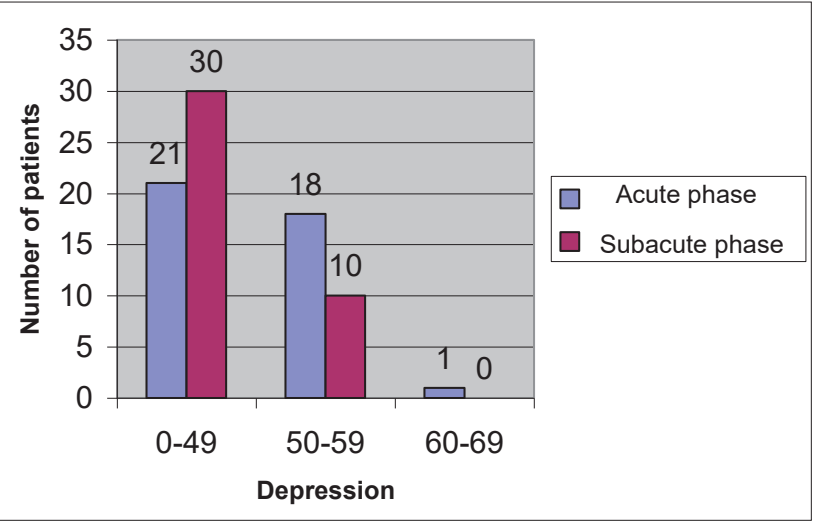

Figure 1. Depression in acute and subacute phase of ischemic stroke. 0-49: normal; 50-59: minor to moderate depression; 6069: moderate to severe depression

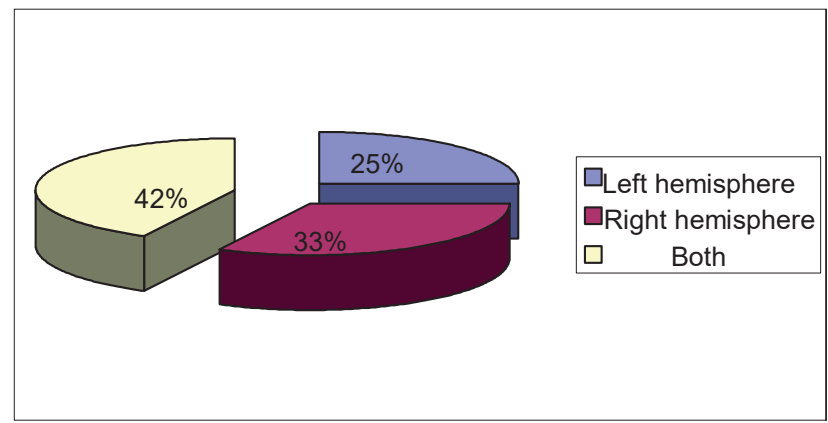

Figure 2. Distribution of ischemic stroke in relation to brain hemisphere

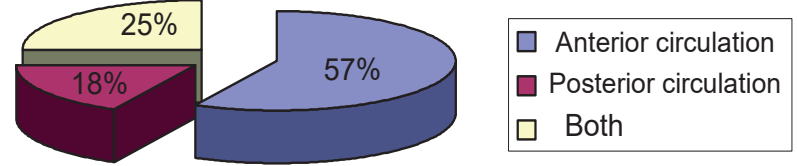

Figure 3. Distribution of ischemic stroke in relation to brain circulation

men $(15 \%)(p=0.06)$.

Mean value on Zung's scales in acute phase of IS in right hemisphere was $47.1 \pm 7.25$ and in left hemisphere $48.8 \pm 10.1$ $(p=0.639)$. The results in subacute phase of IS were: to the right hemisphere $43 \pm 7.5$ and to the left hemisphere $41.9 \pm 6.4$ $(\mathrm{p}=0.71)$ (Figure 2). Concerning the brain circulation (anterior/ posterior), therewere nosignificant differences in meanvalues onZung's scales in acute phase of IS $-47.26 \pm 7.59: 49.42 \pm 11.98$ $(p=0.564)$. Furthermore, in subacute phase results were $44.4 \pm$ 7.7 and $43.4 \pm 9.4(p=0.775)$ (Figure 3).

\section{DISCUSSION}

PSD is defined as a depression that did not exist before the stroke and occurred in chronological context to a stroke. PSD can occur shortly after begin of illness, but is a frequently observed condition in the weeks and months following an acute / subacute phase $(13,14,15)$.

General, patients with PSD have more functional disability, poorer rehabilitation outcomes, and increased morbidity and mortalityin the first year after stroke onset (16). Therefore, it is veryimportanttofindout theriskfactorsforPSD, anddifference between of the clinical presentation some of them.

In our study, number of patients with depression is significantly lower in subacute phase of IS. PSD persisted in the first monthafterISin $25 \%$ ofpatients. Itwassimilartorecentsystematic reviews which indicate the frequency of PSD in about $30 \%$ of survivors $(17,18)$. Interestingly, due to our result, number of patients with PSD is lower in subacute phase of illness and, in regards togender, almost show significant difference $(p=0.06)$. Unfortunately, women are considered to be more likely diagnosedwithsomepsychiatricdisorders, alsoforPSDduringacute and subacute phase of illness (19, 20,21). Oppositely, Ayerbe et al. (2011) failed to find this association (22).

Stroke severity could be one of the most important risk factors for PSD. Movement disorders, dysfunction, and life obstacles caused by brain damage could probably decline the self-confidence of patients. Also, the laterality was one of the firstcharacteristics of IS. Some authors reported frequent links of ischemic lesion in right hemisphere and PSD $(23,24)$. However, there are few studies with opposite findings. Due to their results, damage oflefthemisphereofthebrain, especiallyinthe left frontal lobe and in the left basalganglia. Ithad a close association with the extent of PSD in the acute and subacute phase of illness, persistent / recurrent PSD, as well as poor prognosis after a 1 year time span $(21,25)$. Without exact diagnosis and proper treatment disorder can last up to a year after IS, what 
makes an important impact on the processes of recovery and quality of life (26).

Due to our result, decrease of mean value of depression was observed in patients with IS in posterior circulation of the brain, but not significantly. According to Dennis etal., patients with IS in posterior circulation showbetter emotional recovery (7). Furthermore, there were no differences in mean values of Zung's scales according to brain hemisphere and brain circulation.Similarly,fewsystematicreviewsandmetaanalysissuggest that there is no evident relationship betweenPSD and a specific location of ischemic lesion $(27,28)$.

At present, the neurobiological mechanisms of PSD remain unclear, but the inflammatory factors and other biochemical factors, provide new directions for the prediction of PSD.

PSD is a common problem in daily clinical practice since it is present in 1 out of every 3 stroke patients. More than half of the cases are neither diagnosed nor treated which is a fact that should alert us. In this very unpopular scenario, neurologists play a major role in caring for and managing recovery in stroke patients, and therefore, they must be so familiar with early detection and treatment of PSD (29).

Negative influence of PSD on patient's health is evident. Maybe, the benefit for patients in acute and subacute phase of IS could be higher if PSD would be considered more carefully, followed by an adequate selection and proper plan of medicament and psychotherapeutic approach.

Limitations of our study are: lack of information of stressful life events before IS, sample size, exclusion criteria of speech disorders and neglect (presumably more or less PSD in these two groups of patients), but also absence of comparing our results with severity of IS, age groups as well as level of education. Those are the reasons that justify creation of more comprehensive studies that would overcome current limitations.

\section{CONCLUSION}

Number of patients with PSD is significantly lower in subacute phase of IS. Although the number of depressive women and their depression scores are higher, gender differences are not statistically significant. There is no correlation between PSD and location of lesion in acute and subacute phase of illness.

- Declaration of patient consent: The authors certify that they have obtained all appropriate patient consent forms

- Author's contribution: O.C.I. and Dz.S. gave substantial contributions to the conception or design of the work in acquisition, analysis, or interpretation of data for the work. S.K. had a part in article preparing for drafting or revising it critically for important intellectual content, and each author (O.C.I., Dz. S., S.K., Z. D., A.C., A.S. and B. K. gave final approval of the version to be published and agreed to be accountable for all aspects of the work in ensuring that questions related to the accuracy or integrity of any part of the work are appropriately investigated and resolved.

- Financial support and sponsorship: Nil.

- Conflict of interest: There are no conflicts of interest.

\section{REFERENCES}

1. Anonymous. WHO MONICA Project, Principal Investigators. The World Health Organisation, MONICA Project (monitoring trends and determinants in cardiovascular disease): a major international collaboration. J Clin Epidemiol. 1988; 41: 105-114.

2. European Stroke InitiativeExecutive Committee-EUSIWriting Committee; OlsenTS,LanghorneP,DienerHC,HennericiM,Ferro J,Sivenius J,WahlgrenNG,BathP.EuropeanStrokeInitiativeRecommendationsforStrokeManagement-update2003.Cerebrovasc Dis. 2003; 16(4): 311-337.

3. Kim JS, Choi-Kwon S. Poststroke depression and emotional incontinence: Correlation with lesion location. Neurology. 2000; 54: 1805-1810.

4. Kim JS, Choi-Kwon S, Kwon SU, Lee HJ, Park KA, Seo YS. Factors affectingthequalityoflifeafterischemicstroke:youngversusold patients. J Clin Neurol. 2005; 1: 59-68.

5. Choi-KwonS, KimHS, KwonSU, KimJS. Factorsaffectingtheburden on caregivers of stroke survivors in South Korea. Arch Phys Med Rehabil. 2005; 86: 1043-1048.

6. KimJS. Post-stroke mood and emotional disturbances:Pharmacological therapy based on mechanisms. J Stroke. 2016; 18(3): 244-255.Publishedonline2016Sep30.doi:10.5853/jos.2016.01144

7. Dennis M, O’ Rourke S, Lewis S, Sharpe M, Warlow C. Emotional outcomes after stroke: factors associated with poor outcome. J Neurol Neurosurg Psychiatry. 2000; 68: 47-52.

8. Radulovik-Bekarovska S, Stojanov M, Baneva N, Stefanovski B, Bekarovski N. Post-stroke depression. Book of abstracts of 35th International Danube Symposium for neurological sciencies \& continuing education. Belgrade; 2003: 95-96.

9. Huwel J, Weisner B, Kemmer H, Heyder J. Depressed mood in the acute phase of first ischemic cerebral infarct. Nervenarzt 1998; 69(4): 330-334.

10. ZungWWK.A self rating depression scale. Arch Gen Psychiatry. 1965; 12: 63-70.

11. Agrell B, Dehlin O. Comparison of six depression rating scales in geriatric stroke patients. Stroke. 1989; 20(9): 1190-1194.

12. Domasio C. Cerebralvascular territories. Arch Neurol. 1983;40: 138-142.

13. Nickel A, Thomalla G. Post-stroke depression: Impact of lesion location and methodologicallimitations-A topical review. Front Neurol. 2017; 8:498. Published online 2017 Sep 21. doi: 10.3389/ fneur.2017.00498

14. Carod-Artal FJ. Depresión postictus. Epidemiología, criterios diagnósticos y factores de riesgo. Rev Neurol. 2006; 42: 169-175.

15. Dafer RM, Rao M, Shareef A, Sharma A. Poststroke depression. TopStrokeRehabil.2008;15:13-21.doi:http://dx.doi.org/10.1310/ tsr1501-13.

16. WilliamsLS, GhoseSS, SwindleRW.Depressionand othermental health diagnoses increase mortality risk after ischemic stroke. AmJPsychiatry.2004;161:1090-1095.10.1176/appi.ajp.161.6.1090

17. Goodwin RD, Devenand DP. Stroke, depression, and functional health outcomes among adults in the community. J Geriatr Psychiatry Neurol. 2008; 21(1): 41-46.

18. HackettML,PicklesK.PartI:frequencyofdepressionafterstroke: anupdated systematicreviewandmeta-analysis of observational studies.Int J Stroke. 2014; 9(8): 1017-1025.

19. Bekhbat M. Neigh GN. Sex differences in the neuroimmune consequences of stress: Focus on depression and anxiety. Brain Behav Immun 2017; https://doi.org/10.1016/j.bbi.2017.02.006. Epub ahead of print.

20. Zhang Y, Zhao H, Fang Y, Wang S, Zhou H. The association between lesion location, sex and poststroke depression: Metaanalysis. Brain Behav. 2017; 7(10): e00788. Published online 
2017 Aug 30. doi: 10.1002/brb3.788T

21. Shi Y, Yang D, Zeng Y, Wu W. Risk factors for post-stroke depression: A meta-analysis. Front Aging Neurosci. 2017; 9: 218. Published online 2017 July 11. doi:10.3389/fnagi.2017.00218

22. Ayerbe L, Ayis S, Rudd AG, Heuschmann PU, Wolfe CD. Natural history, predictors, and associations of depression 5 years after stroke: the South London Stroke Register. Stroke. 2011; 421907-1911.10.1161/ STROKEAHA.110.605808.

23. Yu L, Liu CK, Chen JW, Wang SY, Wu YH, Yu SH. Relationship between post-stroke depression and lesion location: a meta-analysis. Kaohsiung J Med Sci. 2004; 20: 372-380. doi:10.1016/S1607-551X(09)70173-1

24. Metoki N, Sugawara N, Hagii J, Saito S, Shiroto H, Tomita $\mathrm{T}$, et al. Relationship between the lesion location of acute ischemic stroke and early depressive symptoms in Japanese patients. Ann Gen Psychiatry. 2016; 15: 12. doi:10.1186/ s12991-016-0099-x

25. Rajashekaran P, Pai K, Thunga R, Unnikrishnan B. Post- stroke depression and lesion location: a hospital based cross-sectional study. Indian J Psychiatry. 2013; 55: 343-348. 10.4103/0019-5545.120546

26. Kronenberg G, Katchanov J, Endres M. Post-stroke depression. Clinical aspects, epidemiology, therapy, and patophysiology. Nervenarzt. 2006; 77(10): 1176-1185.

27. Ayerbe L, Ayis S, Wolfe CD, Rudd AG. Natural history, predictors and outcomes of depression after stroke: Systematic review and meta-analysis. Br J Psychiatry. 2013; 202: 14-21.

28. Kutlubaev MA, Hackett ML. Part II: Predictors of depression after stroke and impact of depression on stroke outcome: An updated systematic review of observational studies. Int J Stroke. 2014; 9: 1026-1036.

29. Espárrago Llorca G, Castilla-Guerra L, Fernández Moreno MC, Ruiz Doblado S, Jiménez Hernández MD. Post-stroke depression: an update. Neurologia. 2015; 30(1): 23-31. doi: 10.1016/j.nrl.2012.06.008. Epub 2012 Aug 16.

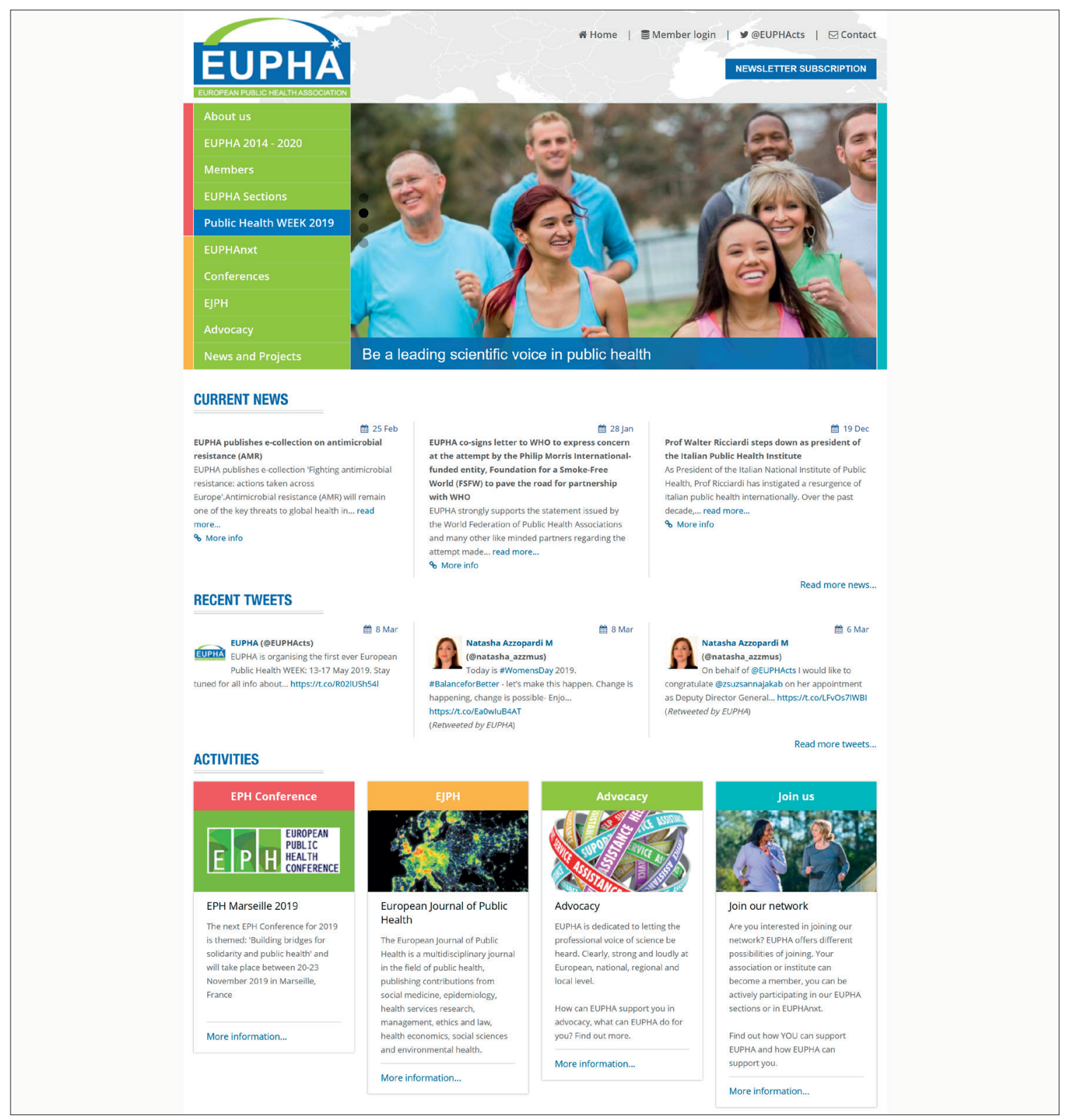

